\title{
The 2017 IUIS Phenotypic Classification for Primary Immunodeficiencies
}

\author{
Aziz Bousfiha $^{1}$ - Leilla Jeddane ${ }^{1,2}$ - Capucine Picard ${ }^{3,4}$ - Fatima Ailal $^{1}$. \\ H. Bobby Gaspar ${ }^{5}$ - Waleed Al-Herz ${ }^{6}$ - Talal Chatila ${ }^{7}$ - Yanick J. Crow ${ }^{8,9}$. \\ Charlotte Cunningham-Rundles $^{10}$ - Amos Etzioni ${ }^{11}$ • Jose Luis Franco ${ }^{12}$. \\ Steven M. Holland ${ }^{13}$ - Christoph Klein ${ }^{14}$ - Tomohiro Morio ${ }^{15}$ - Hans D. Ochs ${ }^{16}$. $^{2}$ \\ Eric Oksenhendler $^{17}$ • Jennifer Puck ${ }^{18}$ • Mimi L. K. Tang ${ }^{19,20,21} \cdot$ Stuart G. Tangye $^{22,23}$ • $^{2}$ \\ Troy R. Torgerson ${ }^{16}$ • Jean-Laurent Casanova ${ }^{24,25,26,27} \cdot$ Kathleen E. Sullivan $^{28}$
}

Received: 26 July 2017 / Accepted: 31 October 2017 / Published online: 11 December 2017

(C) The Author(s) 2017. This article is an open access publication

\begin{abstract}
Since the 1990s, the International Union of Immunological Societies (IUIS) PID expert committee (EC), now called Inborn Errors of Immunity Committee, has published every other year a classification of the inborn errors of
\end{abstract}

Aziz Bousfiha

profbousfiha@gmail.com

1 Laboratory of Clinical Immunology, Inflammation and Allergy LICIA, Faculty of Medicine and Pharmacy, King Hassan II University, Casablanca, Morocco

2 Laboratoire National de Référence, Mohammed VI University of Health Sciences (UM6SS), Casablanca, Morocco

3 Center for the Study of Immunodeficiencies, Necker Hospital for Sick Children, Assistance Publique-Hôpitaux de Paris(APHP), Paris, France

4 Laboratory of Lymphocyte Activation and Susceptibility to EBV, INSERM UMR1163, Imagine Institute, Necker Hospital for Sick Children, Paris Descartes University, Paris, France

5 UCL Great Ormond Street Institute of Child Health, London, UK

6 Department of Pediatrics, Faculty of Medicine, Kuwait University, Kuwait City, Kuwait

7 Division of Immunology, Children's Hospital Boston, Boston, MA, USA

8 Laboratory of Neuroinflammation and Neurogenetics, Necker Branch, INSERM UMR1163, Sorbonne-Paris-Cité, Institut Imagine, Paris Descartes University, Paris, France

9 Division of Evolution and Genomic Sciences, School of Biological Sciences, Faculty of Biology, Medicine and Health, Manchester Academic Health Science Centre, University of Manchester, Manchester, UK immunity. This complete catalog serves as a reference for immunologists and researchers worldwide. However, it was unadapted for clinicians at the bedside. For those, the IUIS PID EC is now publishing a phenotypical classification since 2013,

10

Departments of Medicine and Pediatrics, Mount Sinai School of Medicine, NewYork, NY, USA

11 Ruth's Children's Hospital-Technion, Haifa, Israel

12 Grupo de Inmunodeficiencias Primarias, Facultad de Medicina, Universidad de Antioquia UdeA, Medellin, Colombia

13 Laboratory of Clinical Infectious Diseases, National Institute of Allergy and Infectious Diseases, Bethesda, MD, USA

14 Dr von Hauner Children's Hospital, Ludwig-Maximilians-University Munich, Munich, Germany

15 Department of Pediatrics and Developmental Biology, Tokyo Medical and Dental University (TMDU), Tokyo, Japan

16 Department of Pediatrics, University of Washington and Seattle Children's Research Institute, Seattle, WA, USA

17 Department of Clinical Immunology, Hôpital Saint-Louis, Assistance Publique-Hôpitaux de Paris, University Paris Diderot, Sorbonne Paris Cité, Paris, France

18 Department of Pediatrics, University of California San Francisco and UCSF Benioff Children's Hospital, San Francisco, CA, USA

19 Murdoch Children's Research Institute, Melbourne, VIC, Australia

20 Department of Paediatrics, University of Melbourne, Melbourne, VIC, Australia

21 Department of Allergy and Immunology, Royal Children's Hospital, Melbourne, Australia

22 Immunology Division, Garvan Institute of Medical Research, Darlinghurst, NSW, Australia

23 St Vincent's Clinical School, University of NSW, Sydney, Australia 
which proved to be more user-friendly. There are now 320 single-gene inborn errors of immunity underlying phenotypes as diverse as infection, malignancy, allergy, auto-immunity, and auto-inflammation. We herein propose the revised 2017 phenotypic classification, based on the accompanying 2017 IUIS Inborn Errors of Immunity Committee classification.

Keywords Primary immunodeficiencies · Classification . Phenotypic $\cdot$ IUIS $\cdot$ Inborn errors of immunity

Human primary immunodeficiency diseases (PID) comprise 330 distinct disorders with 320 different gene defects listed [1]. Long considered as rare diseases, recent studies tend to show that they are more common than generally thought, if only by their rapidly increasing number $[2,3]$.The International Union of Immunological Societies (IUIS) PID expert committee proposed a PID classification since 1999 [1], which facilitates clinical research and comparative studies worldwide; it is updated every other year to include new disorders or disease-causing genes. This classification is organized in tables, each of which groups PIDs that share a given pathogenesis. As this catalog is not adapted for use by the clinician at the bedside, the now called Inborn Errors of Immunity Committee proposed since 2013 a phenotypic complement to its classification [4]. Moreover, a smartphone application has been published, based on the 2015 phenotypic classification [5]. As the number of inborn errors of immunity is quickly increasing,
Fig. 1 Immunodeficiencies affecting cellular and humoral immunity. a Severe combined immunodeficiencies defined by T cell lymphopenia. b Combined immunodeficiencies. * T cell lymphopenia in SCID is defined by $\mathrm{CD} 3+\mathrm{T}$ cells $<300 / \mu \mathrm{L}$. AD: autosomal dominant transmission; ADA: adenosine deaminase; $\mathrm{Ag}$ : antigen; $\mathrm{AR}$ : autosomal recessive transmission; $\beta 2 \mathrm{~m}$ : bêta- 2 microglobulin; $\mathrm{Bc}$ : $\mathrm{B}$ cells; $\mathrm{CBC}$ : complete blood count; $\mathrm{CD}$ : cluster of differentiation; CVID: common variable immunodeficiency; def: deficiency; EBV: Epstein Barr virus; HHV8: human herpes virus 8; HIGM: hyper IgM syndrome; HPV: human papillomavirus; Ig: immunoglobulins; MHC: major histocompatibility complex; N1: normal; NK: natural killer; SCID: severe combined immunodeficiency; Tc: T cells; TCR: T cell receptor; Treg: regulatory T cells; XL: X-linked transmission

and at an even faster pace since the advent of next-generation sequencing, this phenotypic classification requires revision at the same pace as the classical IUIS classification.

Here, we present an update of these figures (Figs. 1, 2, $3,4,5,6,7,8$, and 9), based on the accompanying 2017 report in inborn errors of immunity. We included all diseases included in the 2017 update of the IUIS classification [1] and split some categories in two parts to ease the lecture. An algorithm was assigned to each of the nine main groups of the classification and the same color was used for each group of similar conditions. Disease names are presented in red and genes in bold and italics. Mode of inheritance is expressed when adequate; if not expressed, the default mode of transmission is autosomal recessive. Clinical features that point to several diseases are presented in italics before the disease names.
24 St. Giles Laboratory of Human Genetics of Infectious Diseases, Rockefeller Branch, The Rockefeller University, New York, NY, USA

25 Howard Hughes Medical Institute, New York, NY, USA

26 Laboratory of Human Genetics of Infectious Diseases, Necker Branch, INSERM UMR1163, Imagine Institute, Necker Hospital for Sick Children, University Paris Descartes, Paris, France

27 Pediatric Hematology-Immunology Unit, Necker Hospital for Sick Children APHP, Paris, France

28 Division of Allergy Immunology, Department of Pediatrics, The Children's Hospital of Philadelphia, University of Pennsylvania Perelman School of Medicine, Philadelphia, PA, USA 
I. Immunodeficiencies affecting cellular and humoral immunity.

(a) Severe combined immunodeficiencies SCID, defined by CD3 T cell lymphopenia*.

\begin{tabular}{|c|c|c|c|c|c|}
\hline \multicolumn{3}{|c|}{ CD19 NL : SCID T- B+ } & \multicolumn{3}{|c|}{ CD19 $\downarrow:$ SCID T-B- } \\
\hline SCID T-B+NK- & \multicolumn{2}{|c|}{ SCID T-B+NK+ } & SCID T-B-NK- & \multicolumn{2}{|c|}{ SCID T-B-NK+ } \\
\hline $\mathrm{XL}$, & \multirow{2}{*}{$\begin{array}{l}\text { IL7R } \alpha . \\
\text { IL7R }\end{array}$} & \multirow{3}{*}{$\begin{array}{c}\text { Coronin-1A } \\
\text { def . CORO1A } \\
\text { Detectable } \\
\text { thymus }\end{array}$} & ADA def . ADA & \multicolumn{2}{|c|}{ Microcephaly? } \\
\hline CD 132- & & & Chondrosternal & Yes & No \\
\hline $\begin{array}{c}\gamma c \text { deficiency. } \\
\text { IL2RG }\end{array}$ & \multirow{2}{*}{ 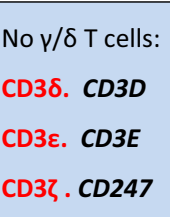 } & & $\begin{array}{l}\text { dysplasia, } \\
\text { deafness, may have }\end{array}$ & $\begin{array}{l}\text { - With facial } \\
\text { dysmorphism: }\end{array}$ & \multirow{4}{*}{$\begin{array}{c}\text { RAG 1/2 def. } \\
\text { (RAG1/ } \\
\text { RAG2) } \\
\text { DCLRE1C def. } \\
\text { DCLRE1C } \\
\text { (ARTEMIS). } \\
\text { Radiosensibi- } \\
\text { lity }\end{array}$} \\
\hline & & \multirow{5}{*}{$\begin{array}{l}\text { Winged helix } \\
\text { def. FOXN1. } \\
\text { Severe } \\
\text { infections; } \\
\text { abnormal } \\
\text { thymic } \\
\text { epithelium; } \\
\text { congenital } \\
\text { alopecia, nail } \\
\text { dystrophy, } \\
\text { neural tube } \\
\text { defect. }\end{array}$} & $\begin{array}{l}\text { pulmonary alveolar } \\
\text { proteinosis, cognitive }\end{array}$ & $\begin{array}{l}\text { DNA ligase IV def. } \\
\text { LIG4 }\end{array}$ & \\
\hline CD 132t & & & & def. NHEJ1. & \\
\hline JAK-3 def. & CD45 def. & & $\begin{array}{l}\text { Reticular dysgenesis. } \\
\qquad \text { AK2 }\end{array}$ & $\begin{array}{l}\text { Radiation } \\
\text { sensitive }\end{array}$ & \\
\hline & PTPRC & & Granulocytopenia, & & \\
\hline & & & $\begin{array}{l}\text { Thrombocytopenia } \\
\text { deafness. }\end{array}$ & $\begin{array}{l}\text { - Without facial } \\
\text { dysmorphism: } \\
\text { DNA PKcs def. } \\
\text { PRKDC } \\
\text { Radiosensibility }\end{array}$ & \\
\hline
\end{tabular}

I. Immunodeficiencies affecting cellular and humoral immunity

(b) Combined Immunodeficiencies Generally Less Profound than Severe Combined Immunodeficiency

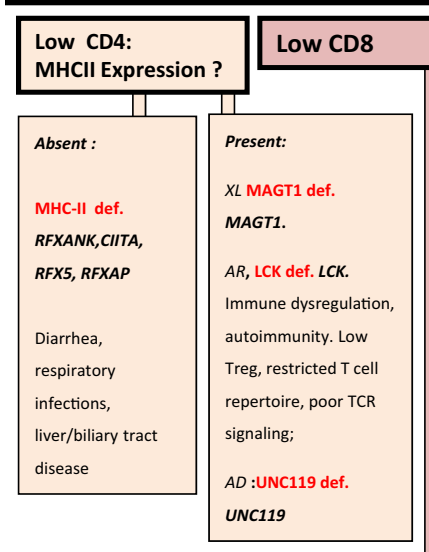

\begin{tabular}{|l|}
\hline CD8 def. CD8A \\
Maybe asymptomatic. CD8 Absent. \\
\hline \hline NI MHCI on lymphocytes. \\
ZAP-70 def. ZAP70 May have immune \\
dysregulation, autoimmunity. NI Ig. CD4: Low \\
fonction
\end{tabular}

\begin{tabular}{|c|c|}
\hline Low Bc: & Ig : often NL \\
\hline \multirow{2}{*}{$\begin{array}{l}\text { DOCK8 def. DOCK8.Severe } \\
\text { Eczema. Cutaneous viral } \\
\text { and staphylococcal } \\
\text { infections; severe atopy; } \\
\text { cancer, diathesis. High IgE, } \\
\text { Low IgM, eosinophilia. Low } \\
\text { NK with poor function. Low } \\
\text { CD27+ memory BC Poor } \\
\text { peripheral Bc tolerance. }\end{array}$} & CD3ץ def. CD3G TCR low. \\
\hline & $\begin{array}{l}\text { RHOH def. } R H O H . H P V \\
\text { infection, lung granulomas, } \\
\text { molluscum contagiosum, } \\
\text { lymphoma. Low naive T } \\
\text { cells, restricted repertoire, } \\
\text { poor proliferation to CD3. }\end{array}$ \\
\hline \multirow{2}{*}{$\begin{array}{l}\text { MST1 def. } \\
\text { STK4.Intermittent } \\
\text { neutropenia; bacterial, viral } \\
\text { (HPV), candidal infections; } \\
\text { EBV Iymphoproliferation; } \\
\text { autoimmune cytopenias; } \\
\text { lymphoma; congenital heart } \\
\text { disease. Low T and B. High } \\
\text { Ig. Low terminal } \\
\text { differentiated effector } \\
\text { memory cells, low naïve Tc, } \\
\text { poor proliferation. }\end{array}$} & $\begin{array}{l}\text { TCR } \alpha \text { def .TRAC. } \\
\text { Recurrent viral, bacterial, } \\
\text { fungal infections; immune } \\
\text { dysregulation and } \\
\text { autoimmunity; diarrhea. } \\
\text { Absent TCR } \alpha \beta \text {; all Tc are } \gamma \delta \text {; } \\
\text { poor proliferation. }\end{array}$ \\
\hline & \multirow{3}{*}{$\begin{array}{l}\text { BCL11B deficiency. } \\
\text { BCL11B. AD. Congenital } \\
\text { abnormalities: neonatal } \\
\text { teeth, dysmorphic facies; } \\
\text { absent corpus callosum; } \\
\text { neurocognitive deficits. TC: } \\
\text { Low, poor proliferation. }\end{array}$} \\
\hline $\begin{array}{l}\text { IL21 def. IL21. Severe early } \\
\text { onset colitis. Low IgG. TC: } \\
\text { NL / low function. }\end{array}$ & \\
\hline \multirow{2}{*}{$\begin{array}{l}\text { NIK def. MAP3K14. } \\
\text { Bacterial, viral and } \\
\text { Cryptosporidium infections. } \\
\text { Low NK and Ig levels. Low } \\
\text { switched memory Bc. Tc :Ag } \\
\text { poor proliferation }\end{array}$} & \\
\hline & $\begin{array}{l}\text { OX40 def. OX40. } \\
\text { Kaposi's sarcoma, impaired } \\
\text { immunity to HHV8. Low }\end{array}$ \\
\hline \multirow[b]{2}{*}{$\begin{array}{l}\text { Moesin def. MSN. XL, } \\
\text { Recurrent infections with } \\
\text { bacteria, varicella; } \\
\text { neutropenia. Low Ig over } \\
\text { time. Tc: defective } \\
\text { migration, proliferation. }\end{array}$} & $\begin{array}{l}\text { memory Bc. Tc : low Ag } \\
\text { specific memory CD4+. }\end{array}$ \\
\hline & $\begin{array}{l}\text { LAT def . LAT. } \\
\text { Adenopathy, } \\
\text { splenomegaly, } \\
\text { autoimmunity. High Ig. } \\
\text { T and B : NL to low }\end{array}$ \\
\hline
\end{tabular}

Normal Ig but Poor Specific Antibody response

function. Poor interferon responses in

hematopoietic and non-hematopoietic cells. IgG NL or low; poor antibody responses.

CARD11 deficiency (LOF). CARD1. Pneumocystis jirovecii pneumonia, bacterial \& viral infections .lg:Absent/low.Tc:NL number, poor proliferation

BCL10 def. BCL10. Recurrent bacterial and viral infections, candidiasis, gastroenteritis. Low memory $\mathrm{T}$ and Treg cells, poor Ag and anti-CD3 prolif. Decreased memory and switched BC

IKBKB def. IKBKB. Recurrent bacterial, viral and fungal infections. Opportunistic infections. Bc: poor fonctions. absent Treg and $\gamma \delta \mathrm{T}$ cells; impaired TCR activation.

ICOS def. ICOS. Autoimmunity, gastroenteritis, granulomas (CVID).

TFRC deficiency. TFRC. Neutropenia, thrombocytopenia. Bc:Nl number, low memory Bc. TC: NI number, poor proliferation.

RelB deficiency.RELB.Tc:poor diversity, poor function

CD40 ligand def. (CD154). XL, CD40LG. or CD40 def. AR, CD40. Opportunistic infections, biliary tract and liver disease, Cryptosporidium. HIGM. Neutropenia, thrombocytopenia, hemolytic anemia, IgM normal or high, other Ig isotypes low. Bc: $\operatorname{slgM} M^{+}, \operatorname{lgD}{ }^{+}$cells present, absent slgG $\mathrm{G}^{+}$, $\operatorname{Ig} \mathrm{A}^{+}$and $\operatorname{lgE} \mathrm{E}^{+}$cells. Tc: NL to low.
IL21R def . IL21R. Recurrent infections;

Pneumocystis, Cryptosporidium Tc: low cytokine production; poor antigen proliferation.

MALT1 def. MALT1. Bacterial, fungal and viral infections. Impaired Tc proliferation and antibody response 


\section{Ila. CID with associated or syndromic features}

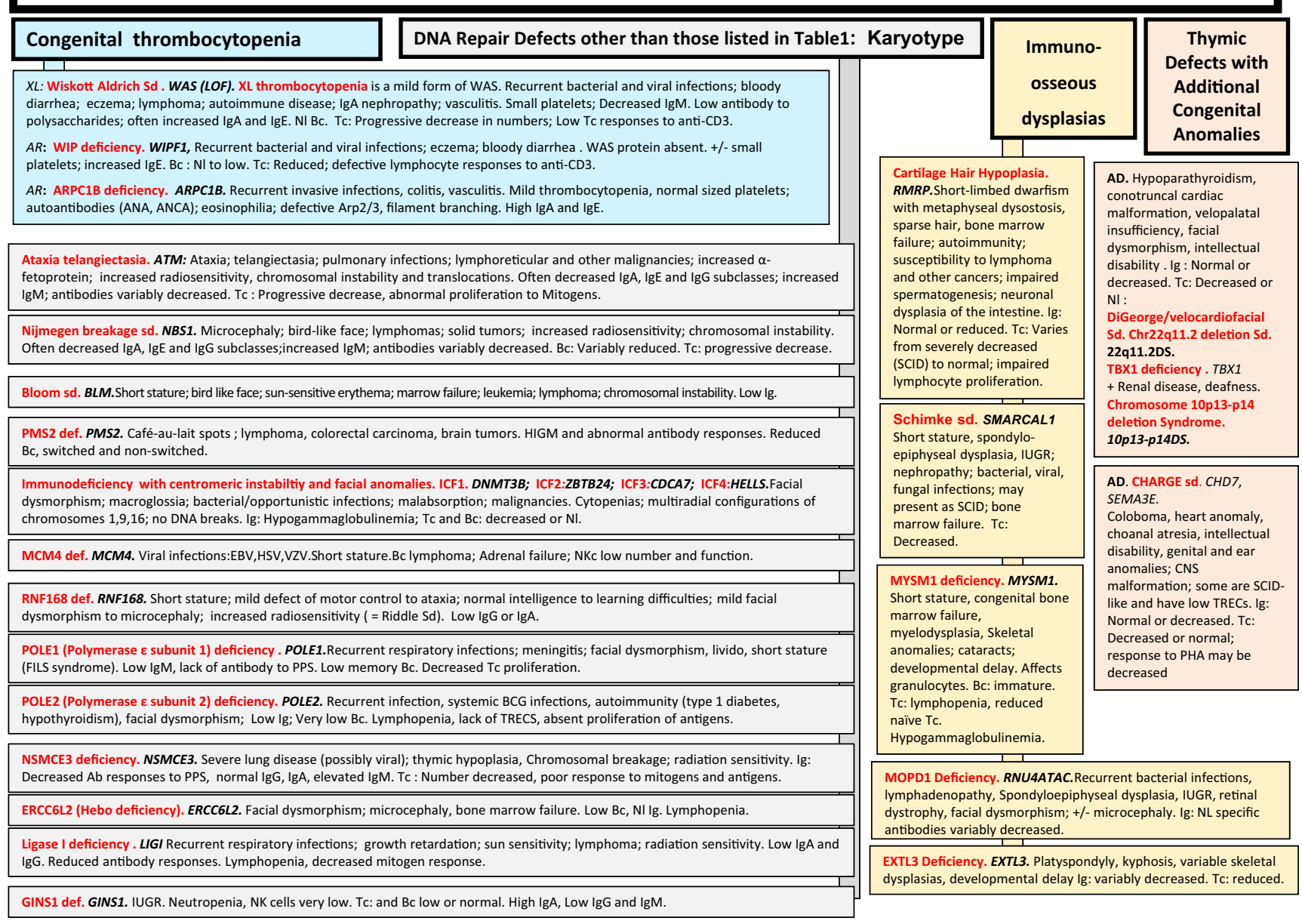

Fig. 2 a, b CID with associated or syndromic features. Ab: antibody; AD: autosomal dominant transmission; ANA: anti-nuclear antibodies; ANCA: anti-neutrophil cytoplasm antibodies; AR: autosomal recessive transmission; Bc: B cells; BCG: Bacillus Calmette-Guerin; BCR: B cell receptor; $\mathrm{CD}$ : cluster of differentiation; CMV: cytomegalovirus; CNS: central nervous system; def: deficiency; DNA: desoxyribonucleic acid; DKC: dyskeratosis congenita; EDA: anhidrotic ectodermal dysplasia; GOF: gain-of-function; HIES: hyper IgE syndrome; FILS: facial dysmorphism, immunodeficiency, livedo and short stature; ID: immunodeficiency; Ig: immunoglobulins; IUGR: intrauterine growth retardation; LOF: loss-of-function; MDS: myelodysplasia; Nl: normal; NK: natural killer; PHA: phytohemagglutinin; PPS: polysaccharides; SCID: severe combined immunodeficiency; sd: syndrome; Tc: T cells; TCR: T cell receptor; TREC: T cell receptor excision circle; XL: X-linked transmission 
IIb. CID with associated or syndromic features

\begin{tabular}{|l|l|}
\hline Hyper-IgE & $\begin{array}{l}\text { Dyskeratosis congenita (DKC) } \\
\text { Syndromes (HIES) }\end{array}$ \\
Myelodysplasia, defective \\
telomere maintenance
\end{tabular}

Defects of Vitamin
B12 and Folate
Metabolism:

\section{Metabolism:}

Megaloblastic anemia, Ig: decreased.

Transcobalamin 2

deficiency. TCN2.

pancytopenia, if

pancytopenia, if
untreated for prolonged

untreated for prolonge
periods results in

intellectual disability.

Deficiency causing

hereditary folate

malabsorbtion. SLC46A1.

if untreated for prolonged

periods results in

intellectual disability

Methylene-

tetrahydrofolate

dehydrogenase

deficiency.

MTHFD1. Recurrent

bacterial infection,

Pneumocystis jirovecii,

neutropenia, seizures,

intellectual disability,

folate-responsive, poor

antibody responses to

conjugated

polysaccharide antigens.

polysacc
Low Bc.

\begin{tabular}{l}
\hline Anhidrotic \\
Ectodermodysplasia \\
with ID
\end{tabular}

\section{Anhidrotic ectodermal}

dysplasia, various infections

(bacteria, mycobacteria, viruses

and fungi), colitis, variable

defects of skin, hair and teeth.

NEMO deficiency. IKBKG

(NEMO). XL, monocyte

dysfunction. Ig decreased,

some with elevated IgA, IgM,

poor specific antibody

responses, absent antibody to

polysaccharide antigens. Bc: $\mathrm{NI}_{\text {, }}$

Low memory and isotype

TCR activation impaired.

EDA-ID due to IKBA GOF

mutation. NFKBIA (IKBA). AD

TC and monocyte dysfunction

elevated IgM, poor specific

antibody responses, absent

antibody to polysaccharide

antigens. Normal Bc numbers,

impar. $B C R$ activation, low,

impaired BCR activation, low

memory and isotype switche

Bc. Normal total Tc, TCR

activation impaired. switched Bc. Tc: NI/decreased,

Decreased $\lg G$ and $\lg A$,

Others

Purine nucleoside phosphorylase deficiency. PNP. Autoimmune hemolytic anemia, neurological impairment. anemia, neurological impairment.
Hypouricemia. Ig : NI/Low. Bc: NI. Tc: Hypouricemia. Ig : NI/L
Progressive decrease

ID with multiple intestinal atresias. TTC7A . Bacterial (sepsis), fungal, viral infections, multiple intestinal atresias, often with intrauterine polyhydramnios and early demise, some with SCID phenotype. Markedly decreased IgG, IgM, IgA. Bc:NI/low.Tc: Variable/absent, low TRECS.

Hepatic veno-occlusive disease with immunodeficiency (VODI). SP110. Hepatic veno-occlusive disease, Pneumocystis jirovecii pneumonia, CMV, candida, thrombocytopenia,

hepatosplenomegaly, cerebrospinal leukodystrophy. Decreased IgG, IgA, IgM, absent germinal centers and tissue plasma cells. Decreased memory BC. Decreased memory Tc.

Vici syndrome. EPG5. Agenesis of the corpus callosum, cataracts,

cardiomyopathy,skin hypopigmentation, intellectual disability, microcephaly, CMC. Ig: Decreased IgG2. Bc: Defective. Profound depletion of $\mathrm{CD} 4+$ cells.

Bacterial infections, autoinflammation, amylopectinosis. Bc: $N I$,decreased memory $B C$. Bacterial infections, autoinflammation, amylopectinosis.Bc: NI, decreased memory
HOIL1 deficiency. HOIL1 (RBCK1). Poor antibody responses to polysaccharides. HOIP deficiency. HOIP1 (RNF31). Lymphangiectasia. Ig: decreased.

Calcium Channel Defects. Autoimmunity, EDA, non-progressive myopathy. Ig and Bc: NI. Tc: Normal, defective TCR mediated activation. ORAI-1 deficiency. ORAl1 . STIM1 deficiency. STIM1

Hennekam-lymphangiectasia-lymphedema syndrome. CCBE1. Lymphangiectasia and lymphedema with facial abnormalities and other dysmorphic features. Ig: decreased. Bc and Tc: Variable.

STAT5b deficiency. STAT5B. Growth-hormone insensitive dwarfism, dysmorphic features, eczema, lymphocytic interstitial pneumonitis, autoimmunity.

Kabuki Sd. Typical facial abnormalities, cleft or high arched palate, skeletal abnormalities, short stature, intellectual disability, congenital heart defects, recurrent infections (otitis media, pneumonia) in $50 \%$ of patients. Autoimmunity may be present. Low IgA and occasionally low IgG. KMT2D (MLL2): AD. KDM6A: XL.

Fig. 2 (continued) 
III. Predominantly Antibody deficiencies, a: Hypogammaglobulinemia

Serum Immunoglobulin Assays : IgG, IgA, IgM, IgE

\section{$\lg G, \lg A$ and/or $\lg M \downarrow \downarrow$}

Exclude second causes: drugs [Hx], myeloma [bone marrow], lymphoma. Ig loss (not hypo-lgM) in urine, gastro-intestinal or skin

$$
\longrightarrow \text { B Lymphocyte (CD19+) enumeration (CMF) }
$$

\begin{tabular}{|l|}
\hline \multicolumn{1}{|c|}{ B absent } \\
\hline \hline Severe bacterial infection. All Ig isotypes \\
decreased. \\
X-Linked Agammaglobulinemia. BTK. \\
Some patients have detectable Ig. ProBc: \\
$\mathrm{NI}$ \\
$A R: \mu$ heavy chain Def. IGHM \\
Ig def. CD79A, Ig $\beta$ def. CD79B \\
BLNK def. BLNK, $\lambda 5$ def. IGLL1 \\
ProBc: NI \\
PI3KR1 def. PIK3R1. ProBc: Decreased \\
AD \\
E47 transcription factor def. TCF3. \\
\hline
\end{tabular}

\begin{tabular}{|c|}
\hline B >1\% \\
\hline Commun Variable Immunodeficiency Phenotype \\
\hline $\begin{array}{l}\text { CVID with no gene defect specified. } \\
\text { Clinical phenotypes vary: most have recurrent infections, some } \\
\text { have polyclonal lymphoproliferation, autoimmune cytopenias } \\
\text { and/or granulomatous disease }\end{array}$ \\
\hline $\begin{array}{l}\text { AD. Severe bacterial infections; EBV susceptibility. } \\
\text { PIK3CD mutation (GOF). PIK3CD GOF. Decreased pro-Bc. } \\
\text { PIK3R1 deficiency (LOF). PIK3CD. Pro-Bc present and low memory Bc. }\end{array}$ \\
\hline $\begin{array}{l}\text { PTEN Deficiency (LOF). PTEN. AD. Lymphoproliferation, } \\
\text { Autoimmunity. }\end{array}$ \\
\hline
\end{tabular}

CD81 deficiency. CD81. Recurrent infections, may have glomerulonephritis.

TACI deficiency. TNFRSF13B (TACI). AD or AR . Variable clinical expression

BAFF receptor deficiency. TNFRSF13C (BAFF-R). Variable clinical expression. Low IgG and IgM.

TWEAK deficiency. TWEAK (TNFSF12). AD. Pneumonia, bacterial infections, warts, thrombocytopenia Neutropenia. Low IgM and A, lack of anti-pneumococcal antibody.

Mannosyl-oligosaccharide glucosidase deficiency (MOGS). MOGS (GCS1). Bacterial and viral infections, severe neurologic disease, also known as congenital disorder of glycosylation type IIb (CDG-Ilb). Severe hypogammagl.

TTC37 deficiency. TTC37. Recurrent bacterial and viral infections, Abnormal hair findings: trichorrhexis nodosa. Poor antibody response to pneumococcal vaccine.

IRF2BP2 deficiency. IRF2BP2. Recurrent infections, possible autoimmunity and inflammatory disease. Hypogammaglobulenia, absent IgA.

CD19 deficiency. CD19. Recurrent infections, may have glomerulonephritis.

CD20 deficiency. CD20. Recurrent infections. Low IgG, NI or elevated IgM and IgA.

CD21 deficiency. Recurrent infections. Low IgG, impaired anti-pneumococcal response.

TRNT1 deficiency. TRNT1. Congenital sideroblastic anemia, deafness, developmental delay. B cell deficiency and hypogammagl.

NFKB1 deficiency. NFKB1. AD. Recurrent sinopulmonary infections, COPD, EBV proliferation, autoimmunity, autoinflammation. Ig normal or low, Bc low or normal, low memory Bc.

NFKB2 deficiency. NFKB2. AD. Recurrent sinopulmonary infections, alopecia and endocrinopathies (ie, central adrenal insufficiency). Low Bc.

IKAROS deficiency. IKZF1. AD. Recurrent sinopulmonary infections. Low or normal Bc potentially reducing levels with age.

ATP6AP1 deficiency. ATP6AP1. XL. Hepatopathy, leukopenia, low copper. Leukopenia and hypogammagl.

\section{Predominantly Antibody deficiencies. b: Other Antibody deficiencies}

\section{Serum Immunoglobulin Assays : IgG, $\lg A, \lg M, \lg E$}

Severe Reduction in Serum IgG and IgA with $\mathrm{NI} /$ elevated IgM and Normal Numbers of BC: Hyper IgM Syndromes

AID deficiency. AICDA.

Bacterial infections, enlarged lymph nodes and germinal centers.

\section{UNG deficiency. UNG.}

Enlarged lymph nodes and germinal centers.

INO80. INO80.

Severe bacterial infections.

\section{MSH6. MSH6.}

Family or personal history of cancer. Variable IgG,

defects, increased IgM in some, NI Bc, low switched

memory Bc.
Isotype, Light Chain, or Functional Deficiencies with Generally NI Numbers of Bc

Selective IgA deficiency. Unknown.

Bacterial infections, autoimmunity mildly increased. Very low to absent IgA with other isotypes normal, normal subclasses and specific antibodies.

Transient hypogammaglobuliemia of infancy. Unknown. Usually not associated with significant infections, normal ability to produce antibodies to vaccine antigens. IgG and IgA decreased.

IgG subclass deficiency with IgA deficiency. Unknown. Recurrent bacterial infections. Reduced IgA with decrease in one or more IgG subclass.

Isolated IgG subclass deficiency. Unknown.

Usually asymptomatic, a minority may have poor antibody response to specific antigens and recurrent viral/bacterial infections. Reduction in one or more $\lg G$ subclass.

Specific antibody deficiency with normal Ig levels and normal B cells. Unknown.

Reduced ability to produce antibodies to specific antigens. Ig: NI.

Ig heavy chain mutations and deletions.

Mutation or chromosomal deletion at $14 q 32$

May be asymptomatic. One or more IgG and/or IgA subclasses as well as IgE may be absent.

Kappa chain deficiency. IGKC

Asymptomatic. All immunoglobulins have lambda light chain.

Selective IgM deficiency. Unknown. Pneumococcal / bacterial

infections. Absent serum IgM.
High $B c$ numbers due to constitutive NF-kB activation

CARD11 GOF

CARD11. AD. BENTA syndrome

Splenomegaly,

lymphadenopathy,

poor vaccine responses. 
Fig. 3 Predominantly antibody deficiencies. a Hypogammaglobulinemias. b Other antibody deficiencies. AD: autosomal dominant transmission; AR: autosomal recessive transmission; Bc: B cells; BENTA: B cell expansion with $\mathrm{NF}-\mathrm{KB}$ and T cell anergy; CD: cluster of differentiation; CMF: flow cytometry; COPD: chronic obstructive pulmonary disease; def: deficiency; EBV: Epstein Barr virus; GOF: gain-of-function; Hx: patient history; Ig: immunoglobulins; Nl: normal; XL: X-linked transmission

\section{Diseases of immune dysregulation. a : Hemophagocytic Lymphohistiocytosis HLH \& EBV susceptibility}

\begin{tabular}{|c|}
\hline Hemophagocytic Lyn \\
\hline $\begin{array}{c}\text { Hypopigmentation: } \\
\text { Partial albinism . Decreased NK and CTL } \\
\text { activities(cytotoxicity and/or degranulation). Bc and } \\
\text { Tc: NI }\end{array}$ \\
\hline $\begin{array}{l}\text { Chediak Higashi sd. LYST } \\
\text { Recurrent infections, fever, (H)SM, bleeding } \\
\text { tendency, progressive neurological } \\
\text { dysfunction. Giant lysosomes (WBC), } \\
\text { neutropenia, cytopenias, Specific hair shaft } \\
\text { anomaly. }\end{array}$ \\
\hline $\begin{array}{l}\text { Griscelli sd type } 2 \text {. RAB27a. } \\
\text { Fever, (H)SM, cytopenias; Specific hair shaft } \\
\text { anomaly. }\end{array}$ \\
\hline $\begin{array}{l}\text { Hermansky Pudlak sd type 2. AP3B1. } \\
\text { Recurrent infections, pulmonary fibrosis, } \\
\text { increased bleeding, neutropenia; Specific hair } \\
\text { shaft anomaly. }\end{array}$ \\
\hline $\begin{array}{l}\text { Hermansky-Pudlak sd, type } 10 . \text { AP3D1. } \\
\text { Oculocutaneous albinism, severe neutropenia, } \\
\text { recurrent infections, seizures, hearing loss and } \\
\text { neurodevelopmental delay. }\end{array}$ \\
\hline
\end{tabular}

Familial Hemophagocytic Lymphohistiocytosis Syndromes: Fever, (H)SM, cytopenias,

NI Bc. Increased activated Tc.

Decreased to absent NK and CTL activities cytotoxicity.

\begin{tabular}{|l|}
\hline Perforin deficiency (FHL2).PRF1. \\
\hline UNC13D / Munc13-4 deficiency (FHL3). \\
UNC13D. \\
\hline
\end{tabular}

Syntaxin 11 deficiency (FHL4). STX11.

STXBP2 / Munc18-2 deficiency (FHL5) STXBP2. Enteropathy
Susceptibility to EBV

\begin{tabular}{|c|c|}
\hline $\begin{array}{l}\text { RASGRP1 deficiency. RASGRP1. } \\
\text { Recurrent pneumonia, herpes virus }\end{array}$ & EBV associated HLH \\
\hline $\begin{array}{l}\text { Increased IgA. Bc and Tc: Poor } \\
\text { activation, proliferation, motility }\end{array}$ & $\begin{array}{l}\mathrm{XL}, \mathrm{XLP1} \text {. SH2DIA. } \\
\text { Clinical and immunologic features }\end{array}$ \\
\hline $\begin{array}{l}\text { CD70 deficiency. CD70 (TNFSF7). } \\
\text { Hodgkin's lymphoma. Reduced IgM, } \\
\text { IgG, IgA ( } 75 \% \text { ) and reduced Ag-specific } \\
\text { Ab responses ( } 50 \%) \text {. Bc:poor antibody } \\
\text { and memory responses. Tc:low Treg, } \\
\text { poor activation and function }\end{array}$ & $\begin{array}{l}\text { lymphoproliferation, Lymphoma. } \\
\text { Hypogamma globulinemia, } \\
\text { Absent iNKT cells. Impaired NK } \\
\text { cell and CTL cytotoxic activity. } \\
\text { Reduced Memory B cells . SAP } \\
\text { deficiency (CMF). }\end{array}$ \\
\hline
\end{tabular}

ation and function

CTPS1 deficiency. CTPS1.

Recurrent/chronic bacterial and viral infections (EBV, VZV), EBV lymphoproliferation, $\quad B C$ non-Hodgkin lymphoma. Tc: poor proliferation to $\mathrm{Ag}$

RLTPR (CARMIL2) deficiency. RLTPR. Recurrent bacterial, fungal and mycobacterial infections, viral warts, molluscum and EBV lymphoproliferative and other malignancy, atopy. Ig NI to low, poor T dependent antibody response. NI Bc. Tc: low Treg, high CD4, poor function.

ITK deficiency. ITK. EBV associated Bc lymphoproliferation, lymphoma, NI or low IgG. Tc: Progressive decrease

MAGT1 deficiency (XMEN). MAGT1.XL. EBV infection, lymphoma, viral infections, respiratory and GI infections. Low $\mathrm{CD} 4$ Low recent thymic emigrant cells, poor proliferation to CD3

PRKCD deficiency. PRKCD. Recurrent infections, EBV chronic infection, lymphoproliferation, SLE-like autoimmunity (nephrotic and antiphospholipid Sd). Low IgG. Low memory $\mathrm{Bc}$ high $\mathrm{CD} 5 \mathrm{BC}$

\section{$\mathrm{XL}, \mathrm{XLP2}$ XIAP.}

Splenomegaly, lymphoproliferation Colitis, IBD, hepatitis. Hypogammaglobulinemia, Low iNKT cells. Increased $T$ cells susceptibility to apoptosis to CD95 and enhanced activation-induced cell death (AICD). Normal NK and CTL cytotoxic activity. XIAP def (CMF)

AR, CD27 deficiency. $\quad$ CD27 (TNFRSF7).

Features triggered by EBV infection, aplastic anemia, low iNKTc lymphoma. Low Ig

FAAP24 deficiency. FAAP24. EBV-driven lymphoproliferative disease. Failure to kill autologous EBV transformed BC.
Fig. 4 Diseases of immune dysregulation. a Hemophagocytic lymphohistiocytosis. b Other diseases of immune dysregulation. Ab: antibody; AD: autosomal dominant transmission; Ag: antigen; ALPS: autoimmune lymphoproliferative syndrome; APS: autoimmune polyendocrinopathy syndrome; $\mathrm{AR}$ : autosomal recessive transmission; $\mathrm{Bc}$ : $\mathrm{B}$ cells; CD: cluster of differentiation; CMF: flow cytometry; CTL: cytotoxic T lymphocytes; def: deficiency; DNT: double negative T cells; EBV: Epstein
Barr virus; FHL: familial hemophagocytic lymphohistiocytosis; GOF: gainof-function; HLH: hemophagocytic lymphohistiocytosis; (H)SM: (hepato)splenomegalia; IBD: inflammatory bowel disease; Ig: immunoglobulin; IL-10: interleukin-10; LOF: loss-of-function; iNKT: invariant NKT cells; NK: natural killer cells; Nl: normal; sd: syndrome; SLE: systemic lupus erythematous disease; Tc: T cells; TCR: T cell receptor; XL: X-linked transmission 


\section{Diseases of immune dysregulation. b: Sd with Autoimmunity and Others}

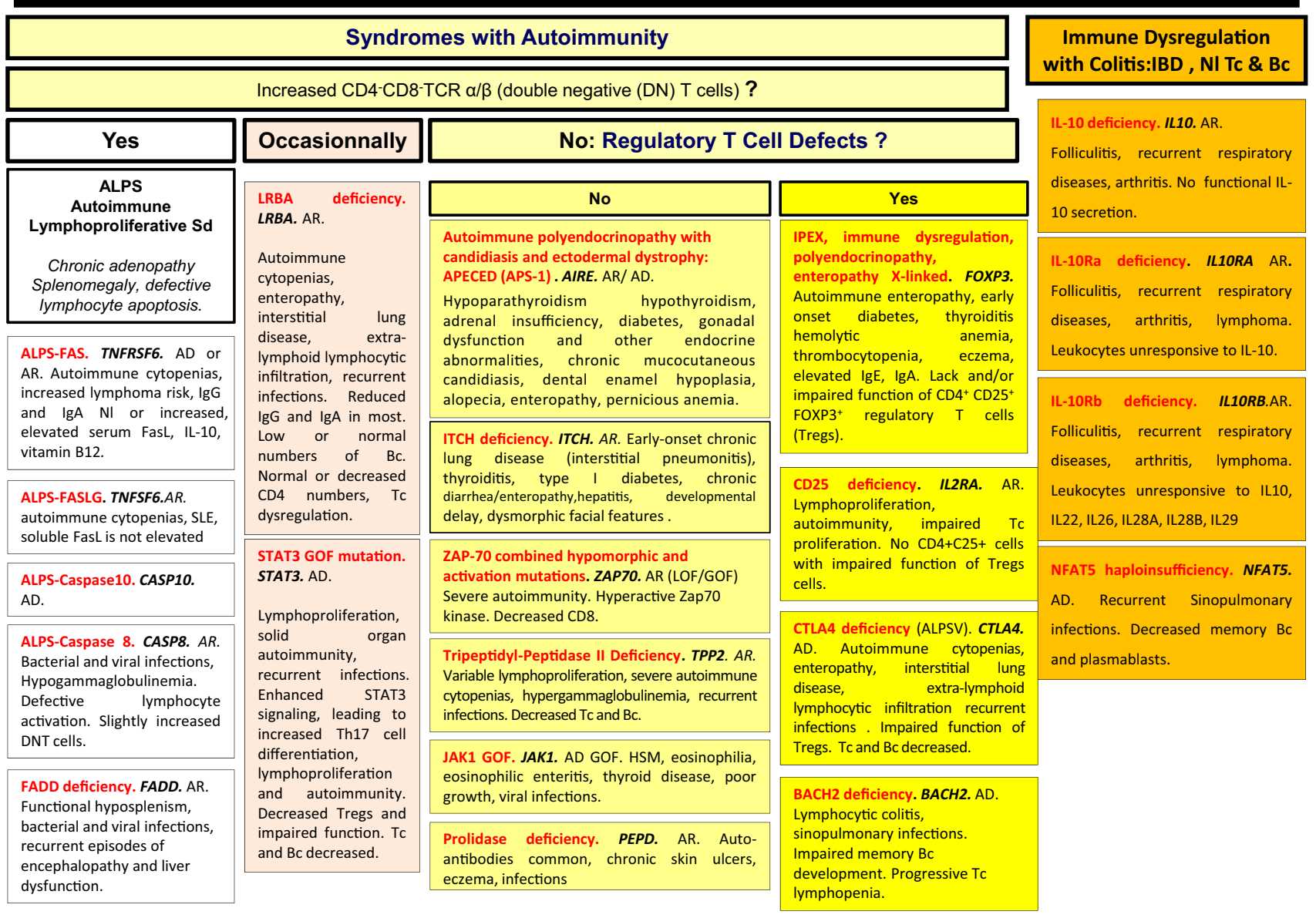

Fig. 4 (continued)

Fig. 5 Congenital defects of phagocyte number, function, or both. a Neutropenia. b Functional defects of phagocytes. AD: autosomal dominant transmission; AML: acute myeloid leukemia; AR: autosomal recessive transmission; $\mathrm{BCG}$ : Bacillus Calmette-Guerin; $\mathrm{CD}$ : cluster of differentiation; CGD: chronic granulomatous disease; CMF: flow cytometry; CMML: chronic myelomonocytic leukemia; def: deficiency; DHR: dihydrorhodamine-1,2,3; GOF: gain-of-function; IUGR: intrauterine growth retardation; MDS: myelodysplasia; NBT: nitroblue of tetrazolium; NK: natural killer cells; WBC: white blood cells; XL: X-linked transmission 


\section{Congenital defects of phagocyte number, function, or both. a : Neutropenia( without anti-PMN)}

\begin{tabular}{|c|}
\hline Syndrome associated \\
\hline $\begin{array}{l}\text { Shwachman-Diamond syndrome. SBDS. AR. DNAJC21. AR. } \\
\text { Pancytopenia, exocrine pancreatic insufficiency, chondrodysplasia }\end{array}$ \\
\hline $\begin{array}{l}\text { G6PC3 deficiency (SCN4). G6PC3. AR. Structural heart defects, urogenital abnormalities, } \\
\text { inner ear deafness, and venous angiectasias of trunks and limbs. Affected functions: } \\
\text { Myeloid differentiation, chemotaxis, } \mathrm{O}_{2} \text { - production. }\end{array}$ \\
\hline $\begin{array}{l}\text { Glycogen storage disease type } 1 \mathrm{~b} \text {. G6PT1. AR. } \\
\text { Fasting hypoglycemia, lactic acidosis, hyperlipidemia, hepatomegaly. }\end{array}$ \\
\hline Cohen syndrome. COH1. AR. Dysmorphism, mental retardation, obesity, deafness. \\
\hline $\begin{array}{l}\text { Barth Syndrome (3-Methylglutaconic aciduria type II). TAZ. XL. } \\
\text { Cardiomyopathy, myopathy, growth retardation. }\end{array}$ \\
\hline $\begin{array}{l}\text { Clericuzio syndrome (Poikiloderma with neutropenia). C160RF57 (USB1). AR. } \\
\text { Retinopathy, developmental delay, facial dysmorphism, poikiloderma. }\end{array}$ \\
\hline $\begin{array}{l}\text { VPS45 deficiency (SCN5). VPS45. AR. } \\
\text { Extramedullary hematopoiesis, bone marrow fibrosis, nephromegaly. }\end{array}$ \\
\hline $\begin{array}{l}\text { P14/LAMTOR2 deficiency. LAMTOR2. AR. } \\
\text { Partial albinism, growth failure. Hypogammaglobulinemia, reduced CD8 cytotoxicity. }\end{array}$ \\
\hline JAGN1 deficiency. JAGN1. AR. Osteopenia. Myeloid maturation arrest. \\
\hline $\begin{array}{l}\text { 3-Methylglutaconic aciduria. CLPB. AR. Neurocognitive developmental aberrations, } \\
\text { microcephaly, hypoglycemia, hypotonia, ataxia, seizures, cataracts, IUGR. }\end{array}$ \\
\hline $\begin{array}{l}\text { SMARCD2 deficiency. SMARCD2. AR. } \\
\text { Developmental aberrations, bones defect, myelodysplasia }\end{array}$ \\
\hline $\begin{array}{l}\text { WDR1 deficiency. WDR1. AR. } \\
\text { Poor wound healing, severe stomatitis, neutrophil nuclei herniate. Mild neutropenia. }\end{array}$ \\
\hline HYOU1 deficiency. HYOU1. AR. Hypoglycemia, inflamn \\
\hline
\end{tabular}

\begin{tabular}{|l|}
\hline \multicolumn{2}{|c|}{ No syndrome associated } \\
\hline \hline Elastase deficiency (SCN1). ELANE. AD. \\
Susceptibility to MDS/leukemia. Severe congenital \\
neutropenia or cyclic neutropenia (perform CBC \\
twice weekly/ 4 weeks). \\
\hline
\end{tabular}

HAX1 deficiency (Kostmann Disease) (SCN3). HAX1. AR. Cognitive and neurological defects in patients with defects in both HAX1 isoforms, susceptibility to MDS/leukemia

\section{GFI 1 deficiency (SCN2). GFI1. AD.}

B/T Iymphopenia

X-linked neutropenia/ myelodysplasia WAS GOF. WAS. Myeloid maturation arrest, monocytopenia, variable lymphoid anomalies .

G-CSF receptor deficiency. CSF3R. AR.

Stress granulopoiesis disturbed

Neutropenia with combined immune deficiency. MKL1. AR.

Mild thrombocytopenia. Lymphopenia.

\section{Congenital defects of phagocyte. $\mathbf{b}$ : Functional defects}

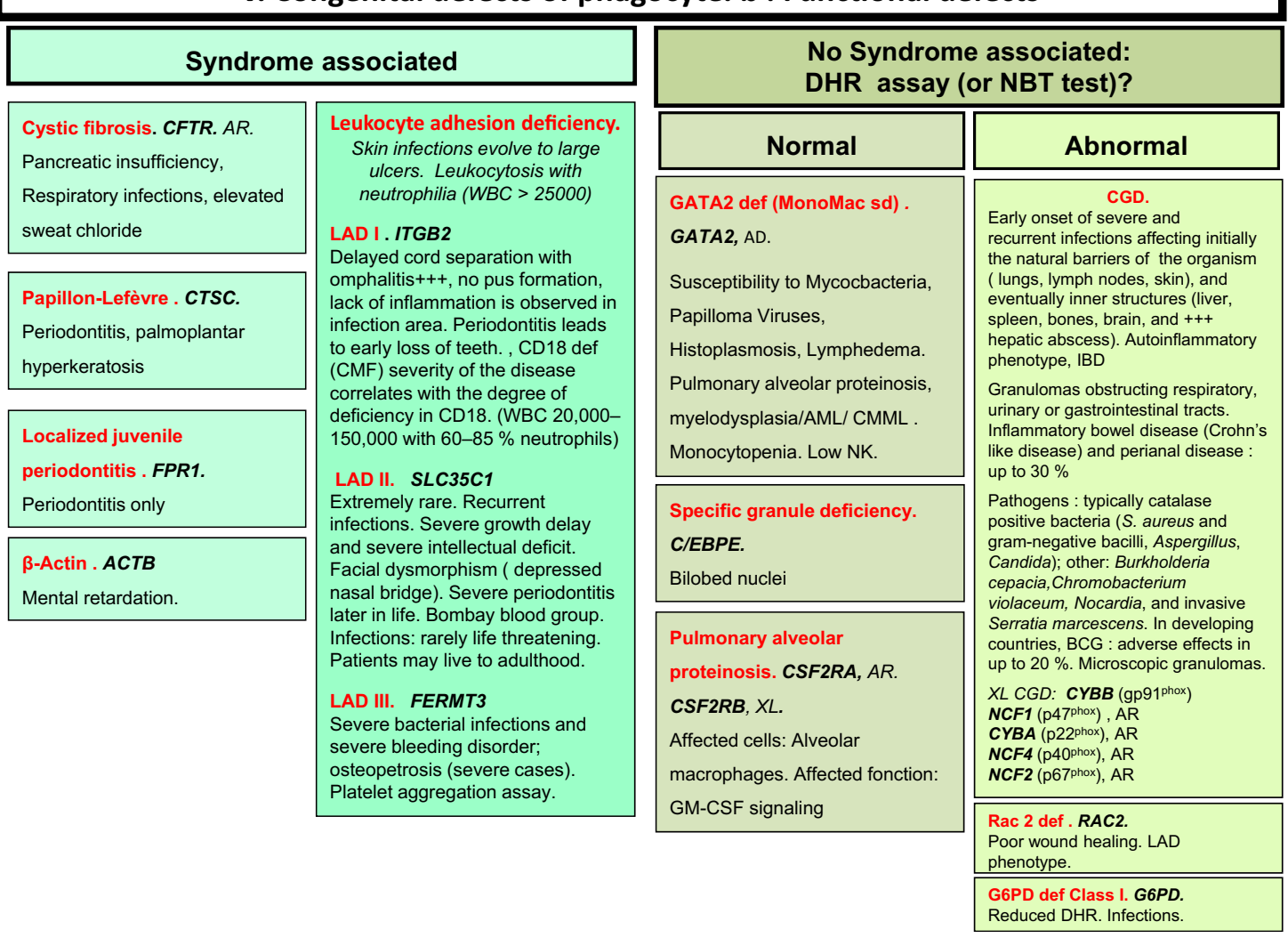




\section{Defects in Intrinsic and Innate immunity. a : Bacterial and Parasitic Infections}

Predisposition to Invasive Bacterial infections
(pyogens):
meningitis, sepsis, arthritis, osteomyelitis and abscesses,
often in the absence of fever.

Predominant pathogens (S. pneumoniae, S. aureus and

Pseudomonas aeruginosa). Non-invasive bacterial infections (skin

infections and upper respiratory tract infections). Improve with age.

Routine Usual screening tests are normal. Specific screening tests

(lack of proinflammatory cytokine production and CD62L shedding) :

available only in specialized clinical immunology laboratories.

IRAK4 def . IRAK4, AR

MyD88 def . MYD88, AR.

IRAK-1 def. IRAK1, XL.

X-linked MECP2 deficiency-related syndrome due to a large de novo

Xq28 chromosomal deletion encompassing both MECP2 and IRAK1

TIRAP def. TIRAP, AR.

Staphylococcal disease during childhood.

Isolated congenital asplenia.

Bacteremia (encapsulated bacteria). No spleen.

RPSA, AD

HMOX, AR. Hemolysis, nephritis, inflammation

\begin{tabular}{|l|}
\hline \multicolumn{1}{|c|}{$\begin{array}{c}\text { Predisposition to Paras } \\
\text { infections }\end{array}$} \\
\hline $\begin{array}{c}\text { Predisposition to Mucocutaneous } \\
\text { Candidiasis (CMC) }\end{array}$ \\
Chronic Mucocutaneous \\
Candidiasis without ectodermal \\
dysplasia
\end{tabular}

\section{STAT1 GOF. STAT1, AD}

various fungal, bacterial and viral (HSV) infections, autoimmunity (thyroiditis, diabetes, cytopenias), enteropathy

\section{IL-17F deficiency. \\ IL17F, $A D$. Folliculitis.}

\section{IL-17RA deficiency.}

IL17RA, AR

Folliculitis. Susceptibility to $S$.

aureus (skin infections)

IL-17RC deficiency.

\section{IL17RC, AR.}

ACT1 deficiency. ACT1, AR.

Blepharitis, folliculitis and

macroglossia.
Fig. 6 Defects in intrinsic and innate immunity. a Bacterial and parasitic infections. b MSMD and viral infection. AD: autosomal dominant transmission; AR: autosomal recessive transmission; BCG: Bacillus Calmette-Guerin; CD: cluster of differentiation; CMC: chronic mucocutaneous candidiasis; GOF: gain-of-function; IFNg: interferon- gamma; HHV6: human herpes virus type 6; HPV: human papilloma virus; HSV: herpes simplex virus; LOF: loss-of-function; MSMD: Mendelian susceptibility to mycobacterial disease; NK: natural killer cells; RNA: ribonucleic acid; sd: syndrome; Tc: T cells; TLR3: Toll-like receptor type 3; VZV: varicella zoster virus; XL: X-linked transmission 


\section{Defects in Intrinsic and Innate immunity. b : MSMD and Viral infection}

\begin{tabular}{|l|l|}
\hline \multicolumn{2}{|c|}{ Mendelian Susceptibility to mycobacterial disease } \\
(MSMD)
\end{tabular}

\begin{tabular}{|c|c|c|}
\hline \multicolumn{3}{|c|}{ Predominant susceptibility to viral infection } \\
\hline Epidermodysplasia & $\begin{array}{l}\text { Predisposition to } \\
\text { Severe Viral Infection }\end{array}$ & $\begin{array}{l}\text { Herpes simplex } \\
\text { Encephalitis. }\end{array}$ \\
\hline \multirow{2}{*}{$\begin{array}{l}\text { EVER1 def. } \\
\text { TMC6.AR. }\end{array}$} & STAT1 Def (AR LOF). & \multirow{5}{*}{$\begin{array}{l}\text { Dominant clinical } \\
\text { phenotype is Herpes } \\
\text { simplex encephalitis (HSE) } \\
\text { during primary infection } \\
\text { with herpes simplex virus } \\
\text { type } 1 \text { (HSV1), usually } \\
\text { between } 3 \text { months and } 6 \\
\text { years of age. Incomplete } \\
\text { clinical penetrance for all } \\
\text { etiologies listed here. } \\
\text { Routine screening tests } \\
\text { are normal. }\end{array}$} \\
\hline & \multirow{2}{*}{$\begin{array}{l}\text { STAT2 deficiency. STAT2. } \\
\text { AR. Disseminated vaccine- } \\
\text { strain measles }\end{array}$} & \\
\hline \multirow{2}{*}{$\begin{array}{l}\text { EVER2 def. } \\
\text { TMC8. AR. }\end{array}$} & & \\
\hline & \multirow{2}{*}{$\begin{array}{l}\text { IRF7 deficiency. IRF7. AR. } \\
\text { Severe influenza disease. } \\
\text { Defect of IFN- } \alpha, \beta \text { and } ~ \\
\text { production and IFN- } \lambda \\
\text { production }\end{array}$} & \\
\hline $\begin{array}{l}\text { WHIM (Warts, } \\
\text { Hypogammaglobuline } \\
\text { mia, infections, myelo- } \\
\text { kathexis) sd. } \\
\text { CXCR4 AD GOF. } \\
\text { Warts (HPV) infection, } \\
\text { neutropenia, low B cell } \\
\text { number, hypogamma- } \\
\text { globulinemia. }\end{array}$ & & \\
\hline \multirow{2}{*}{$\begin{array}{l}\text { kathexis) sd. } \\
\text { CXCR4 AD GOF. } \\
\text { Warts (HPV) infection, } \\
\text { neutropenia, low B cell } \\
\text { number, hypogamma- } \\
\text { globulinemia. }\end{array}$} & $\begin{array}{l}\text { IFNAR2 deficiency. IFNAR2 } \\
\text { AR. Disseminated vaccine- } \\
\text { strain measles, HHV6. No } \\
\text { response to IFN- } \alpha \text {. }\end{array}$ & \multirow{4}{*}{$\begin{array}{l}\text { Specific tests examining } \\
\text { the TLR3 pathway : } \\
\text { marked decrease in the } \\
\text { ability of patient's } \\
\text { fibroblasts to produce IFN- } \\
\alpha \text { and } \beta \text { in response to } \\
\text { HSV1 infection. } \\
\text { TLR3 (AD,AR), } \\
\text { UNC93B1 (AR), TRAF3 } \\
\text { (AD), TICAM1 (TRIF) } \\
\text { (AR,AD), TBK1 (AD), } \\
\text { IRF3 (AD). }\end{array}$} \\
\hline & $\begin{array}{l}\text { CD16 deficiency. FCGR3A. } \\
\text { AR. Severe herpes viral } \\
\text { infections, particularly VZV, } \\
\text { Epstein Barr virus (EBV), } \\
\text { and HPV. }\end{array}$ & \\
\hline & $\begin{array}{l}\text { MDA5 deficiency (LOF). } \\
\text { IFIH1. AR. Rhinovirus and } \\
\text { other RNA viruses }\end{array}$ & \\
\hline & & \\
\hline
\end{tabular}

Fig. 6 (continued) 


\section{VIla. Auto-inflammatory disorders}

Recurrent inflammation Recurrent fever

Familial Mediterranean Fever (FMF) * MEFV. AR or AD

DA: 1-4 days FA : Variable.

Polyserositis, Abdominal pain, Arthritis, Amyloidosis. Erysipelas-like erythema. Predisoposes to vasculitis and inflammatory bowel disease

Colchicine-responsive +++.

Mevalonate kinase def* (Hyper IgD sd). MVK. AR

DA: 3-7 days FA: 1-2 monthly.

Cervical adenopathy. Oral aphtosis. Diarrhea. Mevalonate aciduria during attacks. Leukocytosis with high IgD levels.

TNF receptor-associated periodic syndrome; TRAPS. TNFRSF1A. AD.

\section{DA: 1-4 weeks FA : Variable}

Prolonged fever. Serositis, rash, Periorbital edema and conjunctivitis; Amyloidosis. Joint inflammation.

\section{Systemic inflammation with urticaria rash}

Familial Cold Autoinflammatory Syndrome (CAPS) * . NLRP3, NLRP12. AD GOF DA: $24-48 \mathrm{H}$

Non-pruritic urticaria, arthritis, chills, fever and leukocytosis after cold exposure.

Muckle Wells syndrome (CAPS) *. NLRP3. AD GOF.

Ethnic group : North European

Continuous fever. Often worse in the evenings.

Deafness (SNHL), Conjunctivitis, Amyloidosis.

Neonatal onset multisystem inflammatory disease (NOMID) or chronic infantile neurologic cutaneous and articular syndrome (CINCA) *. NLRP3. AD GOF.

Neonatal onset rash, with continuous fever and inflammation. Aseptic and chronic meningitis, Deforming arthropathy, Mental retardation. Sensorineural deafness. Visual loss.

PLAID (PLCg2 associated antibody deficiency and immune dysregulation), or APLAID*. PLC2G. AD GOF.

Cold Urticaria. Autoimmunity. Blistering skin lesion, pulmonary and bowel disease. Hypogammaglobulinemia, autoinflammation.

NLRP1 deficiency*. NLRP1. AR.

Dyskeratosis, autoimmunity and arthritis.

A20 haploinsufficiency. TNFAIP3. AD LOF. Early onset systemic inflammation, Arthralgia/arthritis, oral/genital ulcers, ocular inflammation.

\section{Others}

CANDLE sd (chronic atypica neutrophilic dermatitis with lipodystrophy).

PSMB8, AR and AD. (Variants in PSMB4, PSMB9, PSMA3, and POMP )

Contractures, panniculitis, ICC, fevers.

COPA defect. COPA. $A D$

Autoimmune inflammatory arthritis and interstitial lung disease with Th17 dysregulation and autoantibody production

NLRC4-MAS (macrophage activating syndrome)*. NLRC4.

AD GOF.

Severe enterocolitis and macrophage activation syndrome (HLH). Triggered by cold exposure.
Fig. 7 a, b Autoinflammatory disorders. *Diseases affecting the inflammasome. AD: autosomal dominant transmission; AR: autosomal recessive transmission; BSN: bilateral striatal necrosis; CAPS: cryopirinassociated periodic syndrome; DA: duration of inflammation episode; FA: frequency of inflammation episode; FCL: familial chilblain lupus; GOF: gain-of-function; HLH: hemophagocytic lymphohistiocytosis;
HSM: hepatosplenomegalia; ICC: intracranial calcifications; IL: interleukin; LOF: loss-of-function; sd: syndrome; SLE: systemic lupus erythematosus; SMS: Singleton-Merten syndrome; SNHL: sensorineural hearing loss; SP: spastic paraparesis; TORCH: toxoplasmosis, other, rubella, cytomegalovirus, and herpes infections 


\section{VIIb. Auto-inflammatory disorders}

\section{Sterile inflammation ( skin / bone / joints )}

Predominant on the bone / joints

Pyogenic sterile arthritis, pyoderma gangrenosum, acne (PAPA) syndrome, hyperzincemia and hyper-

calprotectinemia. PSTPIP1 (C2BP1). AD

DA: 5 days FA: Fixed interval : 4-6 weeks Sterile pyogenic arthritis, Pyoderma gangrenosum, inflammatory skin rash, Myositis. Acute-phase response during attacks

Chronic recurrent multifocal osteomyelitis and congenital dyserythropoietic anemia (Majeed syndrome). LPIN2. AR

\section{DA : Few days FA : 1-3 / month}

Chronic recurrent multifocal osteomyelitis, severe pain, tender soft tissue swelling, Transfusion-dependent anemia, cutaneous inflammatory disorders

DIRA (Deficiency of the Interleukin 1 Receptor Antagonist). ILIRN. AR Continuous inflammation.

Neonatal onset of sterile multifocal osteomyelitis, periostitis and pustulosis.

\section{Cherubism. SH3BP2.}

AR.

Bone degeneration in jaws

Fig. 7 (continued)

\section{Predominant on the skin}

Blau syndrome. NOD2 (CARD15). AD.

Continuous inflammation.

Uveitis, Granulomatous synovitis, Camptodactyly, Rash, Cranial neuropathies, 30\% develop Crohn colitis. Sustained modest acute-phase response.

\section{CAMPS. CARD14. AD. Psoriasis.}

DITRA. (Deficiency of IL-36 receptor antagonist). IL-36RN. AR

Life-threatening, multisystemic inflammatory disease characterized by episodic widespread, pustular psoriasis, malaise, and leukocytosis.

ADAM17 deficiency. ADAM17. AR.

Early-onset pustular dermatitis, short and broken hair, paronychia, frequent cutaneous bacterial infections, and Early onset diarrhea , high IL-1 and IL-6 production. Lack of TNF- $\alpha$ was considered partly responsible for their increased susceptibility to infection and development of cardiomyopathy.

SLC29A3 mutation. SLC29A3 . AR.

Hyperpigmentation hypertrichosis, Rosai-Dorfman like histiocytosis-lymphadenopathy plus $\mathrm{H}$ syndrome

Otulipenia/ORAS. OTULIN. AR.

Arthralgia, Fever, diarrhea, dermatitis. Lipodystrophy, myalgia, Neutrophilia

AP1S3 deficiency. AP1S3. AR. Pustular psoriasis

\section{Type 1 Interferonopathies}

Progressive encephalopathy, ICC, Cerebral atrophy, HSM, leukodystrophy, Thrombocytopenia, Elevated hepatic transaminases . Chronic cerebrospinal fluid (CSF) lymphocytosis

Aicardi-Goutieres syndrome.

TREX1 AR-AD (+SLE, FCL), RNASEH2A, RNASEH2B (+SP), RNASEH2C, SAMHD1 (+ Skin vascularitis, mouth ulcers, arthropathy, FCL), ADAR1 (+BSN, SP), IFIH1 GOF AD (+ SLE, SP, SMS)

Spondyloenchondro-dysplasia dysregulation (SPENCD). ACP5.

Possibly recurrent bacterial and viral infections, SLE-like auto-immunity (Sjögren's syndrome, hypothyroidism, inflammatory myositis, Raynaud's disease and vitiligo), hemolytic anemia, thrombocytopenia, skeletal dysplasia, short stature, SP, ICC.

STING-associated vasculopathy, infantile-onset. TMEM173. Early-onset inflammatory disease, Skin vasculopathy, inflammatory lung disease, systemic autoinflammation and ICC, FCL.

ADA2 deficiency. CECR1. Polyarteritis nodosa, childhoodonset, early-onset recurrent ischemic stroke and fever, Livedo racemosa, low IgM, Hypogammagl, Lymphopenia

$\mathrm{XL}$ reticulate pigmentary disorder. POLA1. Hyperpigmentation, reticulate pattern. Inflammatory lung and Gastroenteritis or colitis. Corneal scarring, characteristic facies

USP18 def . USP18. TORCH like syndrome. 


\begin{tabular}{|c|c|c|c|c|c|}
\hline \multicolumn{6}{|c|}{ VIII. Complement deficiencies } \\
\hline \multicolumn{3}{|c|}{ High } & \multicolumn{3}{|c|}{ Low } \\
\hline \multicolumn{2}{|c|}{$\begin{array}{l}\text { Disseminated } \\
\text { Neisserial infections }\end{array}$} & \multirow{2}{*}{$\begin{array}{l}\text { Recurrent } \\
\text { pyogenic } \\
\text { infections }\end{array}$} & \multirow{2}{*}{$\begin{array}{c}\text { SLE-like syndrome. } \\
\text { Infections with encapsulated } \\
\text { organisms } \\
\text { Absent CH50 hemolytic activity }\end{array}$} & $\begin{array}{l}\text { Atypical Hemolytic Uremic } \\
\text { Syndrome }\end{array}$ & Others \\
\hline Absent $\mathrm{CH} 50$ and $\mathrm{AH} 50$ & Normal CH50. & & & C3 GOF. C3. AD. & C1 inhibitor. \\
\hline $\begin{array}{l}\text { hemolytic activity. } \\
\text { Defective bactericidal } \\
\text { activity. }\end{array}$ & $\begin{array}{l}\text { Absent AH50 } \\
\text { hemolytic } \\
\text { activity }\end{array}$ & \multirow{2}{*}{$\begin{array}{l}\text { C3 LOF. C3. AR. } \\
\text { Absent CH50 and AH50 } \\
\text { hemolytic activity, } \\
\text { defective opsonization } \\
\text { and humoral response }\end{array}$} & C1q def. C1QA, C1QB, C1QC. & $\begin{array}{l}\text { Infections, glomerulonephritis. } \\
\text { Increased activation of complement }\end{array}$ & $\begin{array}{l}\text { SERPING1. } \\
\text { AD, Hereditary }\end{array}$ \\
\hline C5 def. C5 & \multirow{2}{*}{$\begin{array}{l}\text { Properdin } \\
\text { def. } \\
\text { PFC. XL }\end{array}$} & & $\begin{array}{l}\text { C1r def. C1R. Ehlers Danlos } \\
\text { phenotype }\end{array}$ & $\begin{array}{l}\text { Factor B GOF. CFB. AD. Increased } \\
\text { spontaneous AH50 }\end{array}$ & $\begin{array}{l}\text { angioedema. } \\
\text { Spontaneous } \\
\text { activation of the }\end{array}$ \\
\hline C6 def. C6 & & \multirow{2}{*}{$\begin{array}{l}\text { MASP2 def. } \\
\text { MASP2. AR. } \\
\text { Inflammatory lung } \\
\text { disease, autoimmunity }\end{array}$} & \multirow{2}{*}{$\begin{array}{l}\text { C1s def.C1S. } \\
\text { Multiple autoimmune diseases; } \\
\text { Ehlers Danlos phenotype }\end{array}$} & \multirow{2}{*}{$\begin{array}{l}\text { Factor H def. CFH. AR or AD. } \\
\text { Infections, disseminated neisserial } \\
\text { infections, preeclampsia. Spontaneous } \\
\text { activation of the alternative } \\
\text { complement pathway with } \\
\text { consumption of C3 }\end{array}$} & $\begin{array}{l}\text { complement pathway } \\
\text { with consumption of } \\
\text { C4/C2 }\end{array}$ \\
\hline $\begin{array}{l}\text { C7 def. C7. } \\
\text { + Vasculitis }\end{array}$ & $\begin{array}{l}\text { Factor D def. } \\
\text { CFD. AR. }\end{array}$ & & & & \multirow{2}{*}{$\begin{array}{l}\text { Membrane Attack } \\
\text { Complex Inhibitor } \\
\text { deficiency. CD59. } \\
\text { Hemolytic anemia. } \\
\text { Polyneuropathy. }\end{array}$} \\
\hline $\begin{array}{l}\text { C8 def. } \\
\text { C8A, C8B, C8G }\end{array}$ & & $\begin{array}{l}\text { Ficolin } 3 \text { def. } \\
\text { FCN3. AR. } \\
\text { Infections mainly in }\end{array}$ & $\begin{array}{l}\text { C2 def. C2. } \\
\text { Vasculitis, Polymyositis, } \\
\text { atherosclerosis }\end{array}$ & $\begin{array}{l}\text { Factor } \mathrm{H} \text {-related protein } \\
\text { deficiencies. CFHR1-5. AR or AD. } \\
\text { Later onset, disseminated neisserial }\end{array}$ & \\
\hline \multirow{4}{*}{$\begin{array}{l}\text { C9 def. C9. } \\
\text { Mild susceptibility. }\end{array}$} & & $\begin{array}{l}\text { the lungs; abscesses, } \\
\text { necrotizing entero- }\end{array}$ & C4 def.C4A, C4B.AR. & $\begin{array}{l}\text { infections. Normal CH50, AH5O, } \\
\text { autoantibodies to Factor } \mathrm{H} \text {. }\end{array}$ & \multirow{4}{*}{$\begin{array}{l}\text { CD55 deficiency } \\
\text { (CHAPLE disease). } \\
\text { CD55. AR. Protein } \\
\text { losing enteropathy, } \\
\text { thrombosis }\end{array}$} \\
\hline & & $\begin{array}{l}\text { colitis in infancy; } \\
\text { selective antibody } \\
\text { defect to Pneumo- } \\
\text { coccal polysaccharides. } \\
\text { Absence r of } \\
\text { complement activation }\end{array}$ & $\begin{array}{l}\text { C4A or (4B) and appears to have a } \\
\text { modest effect on host defense }\end{array}$ & $\begin{array}{l}\text { Factor I deficiency. AR. Infections, } \\
\text { disseminated neisserial infections, } \\
\text { preeclampsia. Spontaneous activation } \\
\text { of the alternative complement } \\
\text { pathway with consumption of } \mathrm{C3}\end{array}$ & \\
\hline & & $\begin{array}{l}\text { by the Ficolin } 3 \\
\text { pathway }\end{array}$ & & $\begin{array}{l}\text { Thrombomodulin def. THBD. } \\
\text { AD. Normal } \mathrm{CH} 50, \mathrm{AH} 50\end{array}$ & \\
\hline & & $\begin{array}{l}\text { Factor B. CFB LOF. } \\
\text { AR. Infections with } \\
\text { encapsulated } \\
\text { organisms. Deficient } \\
\text { activation of the } \\
\text { alternative pathway }\end{array}$ & & $\begin{array}{l}\text { Membrane Cofactor Protein } \\
\text { deficiency. CD46.AD, Glomerulone- } \\
\text { phritis. Infections, preeclampsia. } \\
\text { Inhibitor of complement alternate } \\
\text { pathway, decreased C3b binding }\end{array}$ & \\
\hline
\end{tabular}

Fig. 8 Complement deficiencies. AD: autosomal dominant transmission; AH50: alternate pathway hemolytic activity; AR: autosomal recessive transmission; CH50: complement hemolytic activity; def: deficiency;
LOF: loss-of-function; sd: syndrome; SLE: systemic lupus erythematosus; XL: X-linked transmission
Fig. 9 Phenocopies of PID. ALPS: autoimmune lymphoproliferative syndrome; AutoAb: auto-antibodies; CID: combined immunodeficiency; CMC: chronic mucocutaneous candidiasis; GOF: gain-offunction; MSMD: Mendelian susceptibility to mycobacterial disease; PRCA: pure red cell aplasia

\begin{tabular}{|c|c|}
\hline \multicolumn{2}{|c|}{ IX. Phenocopies of PID } \\
\hline $\begin{array}{l}\text { Associated with } \\
\text { Somatic Mutations }\end{array}$ & $\begin{array}{l}\text { Associated with } \\
\text { Auto-Antibodies }\end{array}$ \\
\hline \multirow{3}{*}{$\begin{array}{l}\text { Splenomegaly, lymphadenopathy, } \\
\text { autoimmune cytopenias. Defective lymphocyte apoptosis. } \\
\text { ALPS-SFAS. } \\
\text { (somatic mutations in TNFRSF6)/ ALPS-FAS (ALPS type Im) } \\
\text { RALD. RAS-associated autoimmune leukoproliferative } \\
\text { disease. (ALPS Like); N-RAS GOF, K-RAS GOF } \\
\text { Sporadic; granulocytosis, monocytosis/ALPS-like }\end{array}$} & $\begin{array}{l}\text { Chronic mucocutaneous candidiasis (isolated or with APECED } \\
\text { syndrome). AutoAb to IL-17 and/or IL-22. } \\
\text { Endocrinopathy, chronic mucocutaneous candidiasis /CMC. } \\
\text { Germline mutation in AIRE }\end{array}$ \\
\hline & $\begin{array}{l}\text { Adult-onset immunodeficiency with susceptibility to mycobacteria. } \\
\text { Auto-Ab to IFNg. } \\
\text { Mycobacterial, fungal, salmonella, VZV infections / MSMD or CID. }\end{array}$ \\
\hline & \\
\hline \multirow{2}{*}{$\begin{array}{l}\text { Cryopyrinopathy, (Muckle-Wells /CINCA/NOMID-like } \\
\text { syndrome). NRLP3. } \\
\text { Urticaria-like rash, arthropathy, neurological symptoms }\end{array}$} & $\begin{array}{l}\text { Recurrent skin infection. AutoAb to IL-6. } \\
\text { Staphylococcal infections / STAT3 deficiency }\end{array}$ \\
\hline & $\begin{array}{l}\text { Pulmonary alveolar proteinosis . AutoAb to GM-CSF. } \\
\text { Pulmonary alveolar proteinosis, cryptococcal meningitis, }\end{array}$ \\
\hline \multirow{3}{*}{$\begin{array}{l}\text { Hypereosinophilic syndrome due to somatic mutations in } \\
\text { STAT5b. STAT5b. GOF. } \\
\text { Atopic dermatitis, urticarial rash, diarrhea. Eosinophilia. }\end{array}$} & $\begin{array}{l}\text { Acquired angiooedema. AutoAb to } \mathrm{C} 1 \text { inhibitor. } \\
\text { Angioedema / } \mathrm{C} 1 \text { inhibitor deficiency }\end{array}$ \\
\hline & $\begin{array}{l}\text { Atypical Hemolytic Uremic Syndrome. AutoAb to Factor } \mathbf{H} \text {. } \\
\text { Spontaneous activation of the alternative complement pathway }\end{array}$ \\
\hline & $\begin{array}{l}\text { Thymoma with hypogammaglobulinemia (Good syndrome). } \\
\text { AutoAb to various cytokines. Invasive bacterial, viral or } \\
\text { opportunistic infections, autoimmunity, PRCA, lichen planus, } \\
\text { cytopenia, colitis, chronic diarrhea. No B cells. }\end{array}$ \\
\hline
\end{tabular}


Conflict of Interest The authors declare that they have no conflict of interest.

Open Access This article is distributed under the terms of the Creative Commons Attribution 4.0 International License (http:// creativecommons.org/licenses/by/4.0/), which permits unrestricted use, distribution, and reproduction in any medium, provided you give appropriate credit to the original author(s) and the source, provide a link to the Creative Commons license, and indicate if changes were made.

\section{References}

1. Picard C, Gaspar HB, Al-Herz W, Bousfiha A, Chatila T, Crow YJ, et al. International Union of Immunological Societies: 2017 Primary Immunodeficiency Diseases Committee report on inborn errors of immunity. J Clin Immunol 2017(in Press).
2. Bousfiha AA, Jeddane L, Ailal F, Benhsaien I, Mahlaoui N, Casanova JL, et al. Primary immunodeficiency diseases worldwide: more common than generally thought. J Clin Immunol. 2013;33(1): $1-7$.

3. Kobrynski L, Powell RW, Bowen S. Prevalence and morbidity of primary immunodeficiency diseases, United States 2001-2007. J Clin Immunol. 2014;34(8):954-61.

4. Bousfiha AA, Jeddane L, Ailal F, Al Herz W, Conley ME, Cunningham-Rundles $\mathrm{C}$, et al. A phenotypic approach for IUIS PID classification and diagnosis: guidelines for clinicians at the bedside. J Clin Immunol. 2013;33(6):1078-87.

5. Jeddane L, Ouair H, Benhsaien I, El Bakkouri J, Bousfiha AA. Primary immunodeficiency classification on smartphone. J Clin Immunol. 2017;37(1):1-2. 